\title{
Pedestrian Navigation with a Mobile Device: Strategy Use and Environmental Learning
}

\author{
Emily Webber \\ Human Factors Research Group \\ Horizon DTC \\ University of Nottingham, UK \\ psxew@nottingham.ac.uk
}

\author{
Gary Burnett \\ Human Factors Research Group \\ University of Nottingham \\ Nottingham, UK \\ gary.burnett@nottingham.ac.uk
}

\author{
Jeremy Morley \\ Nottingham Geospatial Institute \\ University of Nottingham \\ Nottingham, UK \\ jeremy.morley@nottingham.ac.uk
}

\begin{abstract}
This paper focuses on the strategies employed during a pedestrian navigation task with a mobile device, and the implications for environmental engagement and learning. Twenty-four participants completed a short navigation task using GPS enabled Google Maps on a smart phone. Analysis of verbal protocols and glance behaviour were combined to suggest three broad strategy groups that users fall into when navigating with a mobile device. The results have implications for both environmental learning, and the design of future systems that are sensitive to both context and individual.
\end{abstract}

Navigation; Mobile HCl; Environmental Learning; Individual Differences; Behaviour \& Cognition; Methods

\section{INTRODUCTION}

As the use of mobile navigation aids, such as in-car sat navs and smart phone apps continues to grow, there is a need to be aware of how their use affects our understanding of the environment around us. Previous research suggests that the effects are negative; users become device-focussed and develop a reduced understanding of the environment (e.g. Münzer et al, 2006; Burnett \& Lee, 2005).

Among the most prevalent of mobile mapping applications for pedestrian navigation is Google Maps, which has amassed over 100 million monthly users (Finocchiaro, 2010). This paper describes a study which investigated how navigation and device interaction strategies differ across Google Maps users when completing a short route with a mobile device, and how such differences can influence environmental engagement and learning. By defining and understanding such strategy groups, future mapping applications can be developed which are sensitive to the user, and encourage engagement in the navigation process in synergy with the device that is being used.

\section{BACKGROUND \& RELATED WORKS}

GPS enabled navigation aids mainly provide users with location-based route and survey information. Some devices (particularly in-vehicle) provide timely turn-by turn instruction, whilst other technologies (such as applications on smart phones) provide broader locative route and survey level information. Waters and Winter (2011) discuss three reasons as to the advantages of such devices -1 . There is no longer a need for route planning; 2 . There is no need to maintain one,s orientation whilst travelling, and 3 . Cognitive resources thus are freed for other tasks. Whilst the benefits of these technologies are undeniable reducing stress and increasing the ease and efficiency of navigating to one,s destination - there is growing evidence to suggest that their usage promotes disengagement from the environment (Leshed et al, 2008) and supports only a reduced, understanding of their surroundings (e.g. Münzer et al., 2006; Jackson, 1996).

Recent evidence suggests that spatial learning of an area is negatively affected by the automation caused by, and information received from current navigation systems for both in-car and pedestrian use. Research conducted with drivers found those who used turn-by-turn systems to navigate exhibited a worse memory for an area than those who used notes and paper maps (Burnett \& Lee, 2005), or had no support (e.g. Jackson, 1996). Similar results have been demonstrated for users of pedestrian navigation aids (e.g. Münzer et al., 2006). Encouraging active awareness and engagement with the navigation process and environment, may go some way in reducing the negative effects of navigation automation. Previous studies have gone some way in addressing this by, for example, including additional landmark 
information in the mapping (Oomes, Bojic \& Bazen, 2009), with results supporting increased levels of environmental learning. However, such studies fail to account for differences in the way individuals approach the navigation task, at either a cognitive, motivational or strategic level. Other studies however, have looked at strategy use during navigation with a mobile device. Oulasvirta and colleagues for example, used verbal protocols and strategy interviews to understand how users solved specific problems with mobile maps (Oulasvirta et al, 2005), developing an interesting account of how cognitive processes interact with different aspects of mobile map applications. However, tasks were treated as discrete and didn't take account of the navigation process as a whole, in a naturalistic manner. In addition, as the main aim of the research was to develop a cognitive model, environmental engagement and device requirements were not considered.

The aim of this study is therefore to identify the different strategy archetypes individuals adopt during pedestrian navigation with a mobile device, and the impact these have on environmental engagement and learning. From an understanding of the information requirements of alternative strategy groups, it is anticipated that users can be supported better in the navigation task. Ultimately, this research aims to encourage appropriate device and environment engagement.

\section{METHOD}

The methods reported in this paper form part of a larger study investigating automation levels in pedestrian navigation aids and their effect on environmental learning. The following method description focuses on the specific elements related to the aims of this paper.

\subsection{Setting and Routes}

A quiet residential suburb of Nottingham, UK was selected as the study area (see Figure 1).

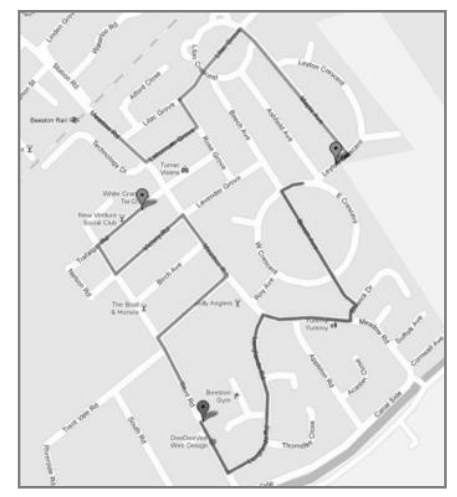

Figure 1: Study Area and Test Routes
Three routes of approximately 0.5 miles each were selected, consisting of five decision points and taking around 10 minutes to complete on foot. Although the routes are close in proximity, it was ensured that a unique vista was observed at the start and finish point of each route so that they were perceived as distinct

\subsection{Participants}

A purposive sample of 24 participants was selected to take part in this study, all of whom reported to be a frequent user of mobile navigation technology (frequent usage was specified as being a minimum of once per month) and were not familiar with the target area either through direct experience, or map use. Selected participants were between 18 and 28 years old (12 male, 12 female; mean age 21.5).

\subsection{Materials}

Participants navigated one of the three pre-defined routes using an HTC Desire running the Google Maps application. The route was highlighted in blue, and a blue arrow showed the users location via GPS. To record both audio and visual, a Veho VCC-003 Muvi Micro DV Camcorder was attached to a headstrap which the participants wore for the duration of the study. Materials and set-up can be seen in Figure 2.
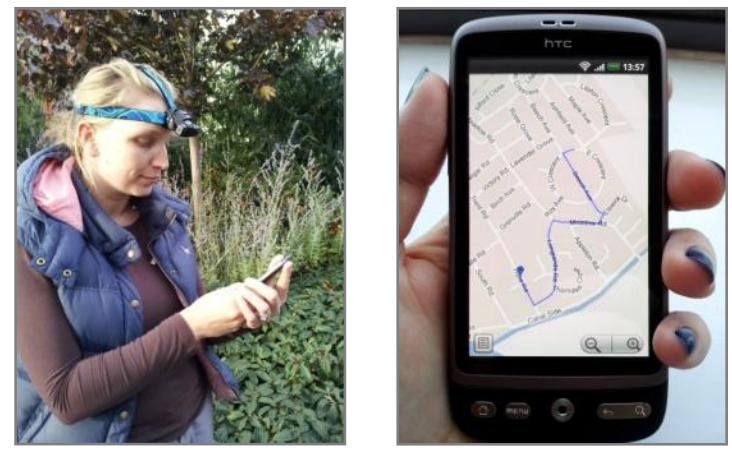

Figure 2: Map interface with route displayed on Google maps on an HTC Desire (Right) \& Material set-up (Left)

\subsection{Procedure}

Participants arrived at a pre-designated meeting point outside the test area, were briefed on the experiment, and familiarised with all technology and procedures. Participants completed one of the three routes using the device, and were asked to stop where they perceived the end point to be. The experimenter followed at a distance of approximately 20 metres to ensure that participants felt as if they were navigating independently, yet were close enough to be informed if they went off route. In such instances, participants were notified of their error within 20 seconds of the deviation, and taken back to their last correct position. As participants navigated each route, they were asked 
to verbalise any task relevant thoughts which came to mind, that were related to either the device and their interaction with it, the surrounding environment, or any relation between the two.

\section{ANALYSIS}

\subsection{Verbal Protocols}

All verbal protocols were transcribed in full. Transcripts from 23 participants were included in the analysis. Data from one participant was excluded, as it was apparent that the aim of the larger study (assessing environmental learning) had been inferred.

To investigate strategy use, a categorisation and coding schema was developed to investigate the types of information utilised from both environment and device, and what function they served in the navigation process. The final method of categorisation was based on two existing schemes; a scheme to identify the information requirements of pedestrian navigation aids (May et. al. 2003), and a taxonomy of useful navigational elements for use within an in-car aid (Burnett, 1998).

The resultant categorisation scheme can be seen in Table 1, and comprises of four different classes of information which could be used at different points within the navigation task. This classification scheme was used to code transcripts in a sequential manner, in order to gain an understanding of how participants used device and environmental information throughout the navigation task. Although the resulting coded data was quantitative in nature, it was decided that a more qualitative assessment of the coded transcripts would be a more suitable method of determining initial strategy groupings. This was decided for two main reasons. Firstly, as an exploration of strategy groups was not an initial aim of the larger study, the use of verbal protocols for this purpose was somewhat post-hoc, and as such, there were no initial hypotheses. Secondly, although the high level of detail that this coding provided gave a complete and detailed insight into information usage and different strategies employed, individual variance meant that the number of potential category combinations became large and unmanageable when combined with the sequential nature of the coding. This meant that individuals varied hugely at such a fine level of detail. Analysis therefore continued in a more qualitative manner. Using the sequential coded transcripts as a starting point, common patterns of information usage were derived - these started on a case by case basis for each participant, and were then generalised to form archetypal strategy groups which vary in terms of the information provisions required by the user, particularly in terms of the source, location, and usage categories.

Table 1: Classification of information used within a pedestrian navigation task

\begin{tabular}{|l|l|}
\hline Primary category & Secondary categories \\
\hline Source & $\begin{array}{l}\text { Environment } \\
\text { Device }\end{array}$ \\
\hline $\begin{array}{l}\text { Location (At which } \\
\text { required) }\end{array}$ & $\begin{array}{l}\text { Path } \\
\text { Node }\end{array}$ \\
\hline Usage & Preview (next or future) \\
& Identify \\
& Confirm \\
& SWYS (say what you see \\
& - no navigational function) \\
\hline Type & Landmark \\
& Street/Sign name \\
& GPS \\
& Direction \\
& Distance \\
& Road/Junction \\
\hline
\end{tabular}

\subsection{Attention maps}

Glance behaviour was analysed to form a timeline of device glances. Figure 3 depicts an example of an attention map for one participant completing one route. The attention map runs left to right, totalling the number of seconds taken to complete the navigation task. Each black dot represents a typical ,short' glance (with a mean duration of 3.4 seconds) towards the mobile device. Longer glances (over 6 seconds) are highlighted by a black bar that extends from each glance dot, representing the total glance duration. As the participant moves through the route, each decision point encountered is represented by a solid red line. The pale red indicates the point in time at which that junction becomes identifiable in the environment. The blue lines highlight any other points of navigational uncertainty; these junctions which are passed on the left and right, appear at the top and bottom of the map respectively. Each attention map can be thought of as a unique visualisation of each participant moving through the route - the black representing when they are attending to the device, the spaces between, the environment.

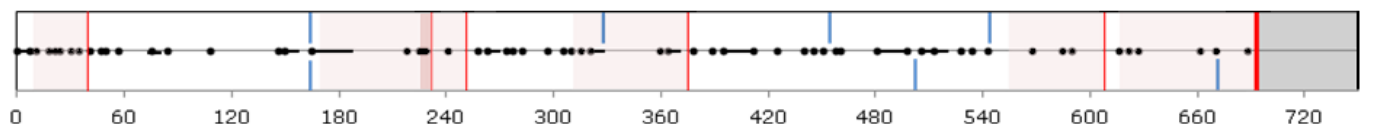

Figure 3: Example Attention Map 


\section{RESULTS AND DISCUSSION}

Table 2: Overview of Strategy groups for pedestrian navigation with a mobile aid

\begin{tabular}{|c|c|c|}
\hline $\begin{array}{l}\text { Strategy } \\
\text { group name }\end{array}$ & General Description & $\begin{array}{l}\text { Implications for the provision of information to } \\
\text { encourage environmental engagement }\end{array}$ \\
\hline $\begin{array}{l}\text { Constant } \\
\text { support \& } \\
\text { Information }\end{array}$ & $\begin{array}{l}\text { - Constant support \& information from both } \\
\text { environment and device. } \\
\text { - Information sought at all stages of route } \\
\text { - More information (regardless of when \& } \\
\text { what - i.e. not necessarily strategic or } \\
\text { tactical) is better. }\end{array}$ & $\begin{array}{l}\text { - Information should be accessible at regular } \\
\text { intervals. Increase amount of information with } \\
\text { each successive map presentation for points of } \\
\text { uncertainty along path sections \& nodes } \\
\text { Information push initially to support confidence } \\
\text { building, then gradually reduce and move to } \\
\text { user controlled } \\
\text { Aid given in what to look for to confirm route - } \\
\text { e.g. a landmark image or street name. }\end{array}$ \\
\hline $\begin{array}{l}\text { Independent } \\
\text { \& Attentive }\end{array}$ & $\begin{array}{l}\text { - Navigators are actively engaged in the } \\
\text { navigation task \& with the environment. } \\
\text { - The device is used for tactical information } \\
\text { retrieval for decisions regarding nodes } \\
\text { and salient points along the path. } \\
\text { - Users may exhibit forward planning (past } \\
\text { the next decision point), and increased } \\
\text { landmark identification }\end{array}$ & $\begin{array}{l}\text { - Minimal information - enough to support task at } \\
\text { key points } \\
\text { - } \quad \text { No information push, user controlled } \\
\text { Accessible overview mode to aid forward } \\
\text { planning \& development of survey knowledge } \\
\text { - Correctly mapped landmarks to maintain device } \\
\text { confidence. }\end{array}$ \\
\hline $\begin{array}{l}\text { Least Effort \& } \\
\text { Inattentive }\end{array}$ & $\begin{array}{l}\text { - Navigators exhibit minimal effort and } \\
\text { engagement during navigation with } \\
\text { device } \\
\text { - Would rather rely on GPS to guide them. } \\
\text { - Environmental referents only used in } \\
\text { conjunction with GPS to confirm location } \\
\text { and occasionally identify turnings for } \\
\text { clarity. }\end{array}$ & $\begin{array}{l}\text { - Minimise reliance by including info which } \\
\text { - } \quad \text { Pushes attention from device into environment } \\
\text { attention to key environmental features. } \\
\text { - Decrease constant GPS support - users to } \\
\text { "ask" for location information. }\end{array}$ \\
\hline
\end{tabular}

Results have been collapsed across the three routes in which participants used Google Maps with route information and GPS to navigate.

Table 2 gives an overview of the three different strategy groups that emerged from qualitative analysis of the sequentially coded verbal protocols. It also highlights the implications for the provision of information to users in a way that would support individual differences in navigational style, as well as encouraging environmental engagement. Although the table describes each of these strategy groups as discrete, in reality, this is not necessarily the case. Individuals will fluidly shift between groups as changes in context, personal and situational needs dictate. However, it is likely that a particular user will generally fall into one of these categories during a typical navigation situation.

The implications for the provision of information were derived from consideration of how information from device and environment is used, and the potential impact this has on environmental engagement, and learning. Take, for example, those in the Constant Support and Information group. Their need for information is high at all stages of navigation, with constant repetition of route level information highlighting the need for continual confirmation and support. Confirmation between information from device and environment is high and continual, with GPS providing an additional moment-by-moment means of location confirmation. Contrastingly, those in the Independent and Attentive group have far fewer device interactions, but the ones they have, are meaningful and targeted, referring to key points on the route (points of uncertainty and decision points). Users will engage in planning activities, often long before the they reach the point in the route, memorising important reference points for later use. Additionally, users will often refer to their global orientation and identify shortcuts in the routes.

Archetypal interaction strategies such as those described, can thus be used to create recommendations for the provision of navigational information in a way that supports the user in the task, and encourages engagement with the environment in a meaningful manner.

However, there are limitations to using verbal protocols in this way. Firstly, verbalising cognitive processes has been shown to influence the way participants approach a particular task (see Russo, Johnson \& Stephens, 1989, for a review) and can 

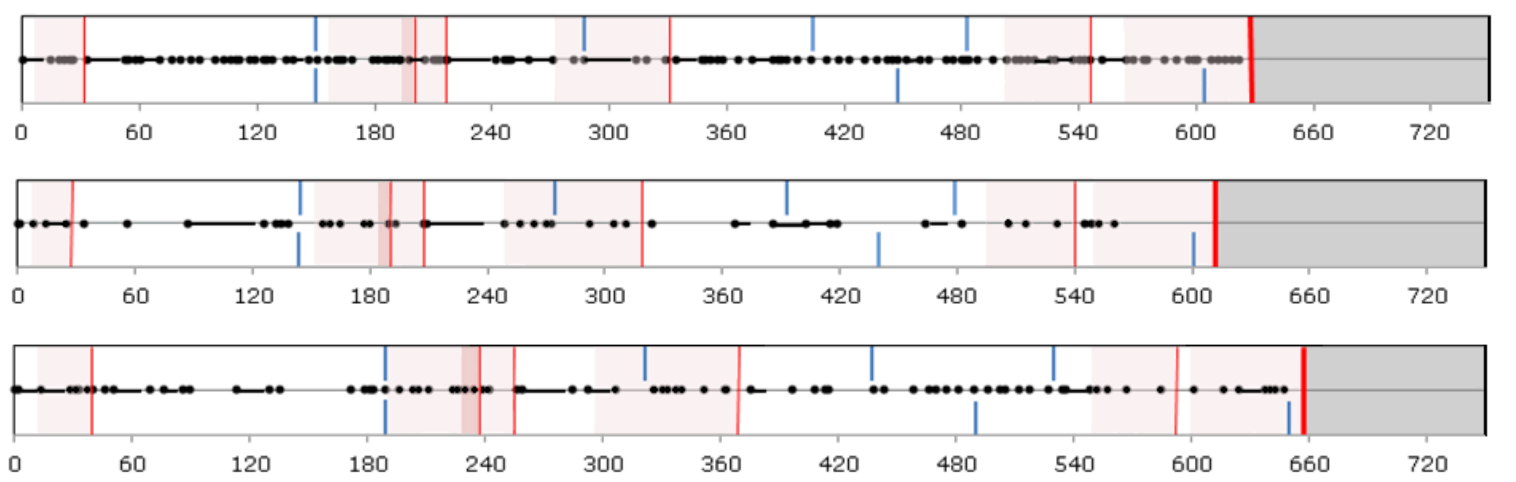

Figure 4: Example attention maps for each of the three strategy groups. From top to bottom; Constant Support (P4), Independent \& Attentive (P11) and Least Effort \& Inattentive (P22)

change both their cognising and subsequent actions. Secondly, verbalisation requires a degree of cognitive effort. As tasks become increasingly difficult, participants are therefore likely to reduce the number of utterances, to focus on the main task. In a navigation context, this might result in a reduction in verbalisations during planning phases, for example, or during periods of navigational uncertainty. However, verbal protocols have been used within a navigation context before (e.g. Kato \& Takeuchi, 2003), showing them to be a valid method of extracting task related cognitive thought. The strategy groups derived from verbal protocols form an initial starting point, and therefore require validation from other sources.

Analysis of glance behaviour provides a means of confirming some of the key strategy group indicators that were derived from the verbal protocols. Attention maps depict patterns of glances which can highlight key interaction and navigation strategies such as those described in Table 2. For example, individuals who fall into the Constant Support and Information strategy group would be likely to show indiscriminate and frequent glances to the device. Contrastingly, those in the Independent Navigation group might show targeted glances around points of navigational uncertainty, and longer or more frequent glances at key points which might indicate planning. While such types of glances may equally be indicative of confusion, also resulting in more time spent looking at a device at a particular location, consideration of the coded verbal protocols that accompany the glance pattern can confirm the type of glance that occurred. It is clear then, that consideration of the attention maps without the corresponding verbal protocols reveals little about how these glance patterns relate to strategy use, as they do not contain information about the underlying cognitive processes and motivations.

Figure 4 highlights the attention maps of three participants who might fall into each of the initial strategy groups. At a glance, the differences between these groups may at first seem arbitrary, yet taken with the verbal protocols, they reveal how and why particular users might be interacting with both device and environment. For example, Participant 4 can be said to fall into the Constant Support and Information group. Glances occur frequently and at regular intervals, with device time outweighing the time spent looking at the environment. Verbal protocols reveal that glances and interactions are repetitive in nature, searching for and confirming the same information multiple times, with very little difference in location or available information. Conversely, the attention map of Participant 11, who falls into the Independent and Attentive group, reveals fewer glances. These occur mainly at targeted locations for example, identifying or confirming a junction in the environment. Consideration of the corresponding verbal protocol reveals that longer glances are usually planning type interactions, with this information either being committed to memory and used at a later stage or used to gain an understanding of the entire route, and their orientation within it

Participants falling into the Least Effort and Inattentive group are harder to identify, as device interaction differs hugely between individuals and appears generally arbitrary in terms of their location, type, and purpose. Although verbal protocols reveal glance patterns to be typified by confirmatory glances to the GPS marker as a constant location update, and use few environmental referents, it is difficult to see confirmation of these types of patterns through the attention maps. Perhaps there is something more subtle regarding the way in which glances move between device and environment which is suggestive of less engagement and decreased attention to the environmental referents which are central to the navigation task, which is missed through use of these methods. It is clear then, that further work remains to be conducted on these 
strategy groups, particularly for the latter „inattentive' group. Such work would provide further validation and grounding for the preliminary findings discussed here. Although an initial investigation, the combination of the two methods discussed here provides a strong foundation from which to base such future research.

\section{CONCLUSIONS AND FUTURE RESEARCH}

Taken together, consideration of the verbal protocols and attention maps opens a window into the types of strategies that users adopt when navigating with pedestrian mobile aids. Three main strategy groups have emerged - users who are Independent and Attentive in the navigation task, those who need Constant Support and Information, and those who give Least Effort and are Inattentive. Though these groups provide an initial outline of these different strategies, it is important then, to understand the reasons why these differences occur. For example, groups may vary with particular individual traits, such as navigational confidence or perceived sense of direction, along a conceptual continuum. However, this change may not be as simple as a change in one particular individual trait, but as a result of a network of interactions between them, and other factors. It is important to understand the reasons why individuals vary in terms of strategies, in order to provide them the appropriate support, offering new strategies and methods for both navigation and environmental engagement.

Although the definition and understanding of processes and motivations inherent within each of the groups is at an early stage, they offer a starting point from which to build on in future research. Such research could offer triangulation of evidence from eye-tracking, which could confirm the finer patterns of device and environmental interaction with quantitative data. Given the limitations of this study, as discussed above, it is important that the evidence for such groups is derived from a variety of sources and methodologies, to triangulate evidence and provide support for such a strategy framework. By understanding how the use of strategy varies across users, we can further detail the provision of information requirements necessary to support the user in the navigation task, whilst encouraging meaningful engagement with the surrounding environment.

\section{ACKNOWLEDGEMENTS}

The first author is supported by the Horizon Doctoral Training Centre at the University of Nottingham (RCUK Grant No. EP/G037574/1). Thank you to Alpkit for kindly donating the headstraps used within this study.

\section{REFERENCES}

Burnett, G. (1998) „,Turn right at the Kings Head": drivers' requirements for route guidance information. PhD Dissertation, Loughborough University

Burnett, G. \& Lee, K. (2005). The effect of vehicle navigation systems on the formation of cognitive maps, In Underwood, G. (ed.)Traffic and Transport Psychology: Theory and Application , 407-418, Elsevier: Amsterdam

Finocciaro, P. (2010) Google Maps for mobile claims $100 \mathrm{M}$ monthly users http://bit.ly/amDope (retrieved May 2012)

Forlizzi ,J., Barley, W., \& Sedar, T. (2010) Where Should I Turn? Moving from Individual to Collaborative Navigation Strategies to Inform the Interaction Design of Future Navigation Strategies. In Proc.CHI '10, Atlanta, 10-15 April '10,1261-1270

Jackson, P. (1996) How will route guidance information affect cognitive maps? Journal of Navigation. 49(2), 178-186

Kato, Y., \& Takeuchi, Y. (2003) Individual differences in wayfinding strategies. Journal of Environmental Psychology, 23(2),171 - 188

Leshed, G., Velden, T., Rieger, O., Kot, B., \& Sengers, S. (2008) In-Car GPS Navigation: Engagement with and Disengagement from the Environment. In Proc CHI '08, Florence, 5-10 April '08, 1675-1684

May, A., Ross, T., Bayer, S. \& Tarkianinen M. (2003) Pedestrian Navigation Aids: Information Requirements and Design Implications. Pervasive Ubiquitous Computing, 7(6), 331-338

Münzer, S., Zimmer, H., Schwalm, M., Baus, J. \& Aslan, I. (2006) Computer-assisted navigation and the acquisition of route and survey knowledge. Journal of Experimental Psychology 26(4) 300-308

Oomes, A.H., Bojic, M. \& Bazen, G. (2009)

Supporting Cognitive Collage Creation for

Pedestrian Navigation. In Harris, D. (ed.)

Engineering Psychology and Cognitive

Ergonomics, HCII 2009. LNCS (LNAI), vol. 5639, 111-119. Springer, Heidelberg

Oulasvirta, A., Nivala, A. M., Tikka, V., Liikkanen, L., \& Nurminen, A. (2005). Understanding users strategies with mobile maps. In Proc. Mobile $\mathrm{HCl}$ '05, Salzburg, 19 Sept '05, 1-6

Russo, J., Johnson, E. \& Stephens, D. (1989) The Validity of Verbal Protocols. Memory \& Cognition. 17 (6), 759-769.

Waters, W. \& Winters, S. (2010) A Wayfinding aid to increase navigator independence. Journal of Spatial Information Science 2(1) pp 1-17 